doi: $10.15407 /$ ujpe62.06.0533

M.V. USHCATS, ${ }^{1,2}$ L.A. BULAVIN, ${ }^{1}$ V.M. SYSOEV ${ }^{1}$ S.YU. USHCATS ${ }^{2}$

1 Taras Shevchenko National University of Kyiv

(2, Prosp. Academician Glushkov, Kyiv 03680, Ukraine)

2 Admiral Makarov National University of Shipbuilding

(9, Heroes of Ukraine Ave., Mykolaiv 54025, Ukraine; e-mail: mykhailo.ushcats@nuos.edu.ua)

PACS 05.20.-y, 05.20.Jj, 05.50.+q, 05.70.Ce, 51.30. $+\mathrm{i}, 64.10 .+\mathrm{h}$, 64.60.De

\section{LATTICE GAS CONDENSATION AND ITS RELATION TO THE DIVERGENCE OF VIRIAL EXPANSIONS IN THE POWERS OF ACTIVITY}

\begin{abstract}
An efficient algorithm for the calculation of high-order reducible cluster integrals on the basis of irreducible integrals (virial coefficients) has been proposed. The algorithm is applied to study the behavior of the well-known virial expansions of the pressure and density in power series of activity up to very high-order terms, as well as recently derived symmetric power expansions in the reciprocal activity, in the framework of a specific lattice gas model. Our results are consistent with those obtained in other modern studies of the partition function in terms of the density. They disclose the physical meaning of the divergence that the mentioned expansions demonstrate in the condensation region.
\end{abstract}

Keywords: lattice gas, virial coefficients, reducible cluster integrals, activity, equation of state, condensation.

\section{Introduction}

A new statistical approach has been proposed recently [1-3] for a wide range of lattice gas models, in which the "particle-hole" symmetry inherent in such models is used. For the general well-known virial equation of state (VEOS) $[4,5]$

$\frac{P}{k_{\mathrm{B}} T}=\rho\left(1-\sum_{k \geq 1} \frac{k}{k+1} \beta_{k} \rho^{k}\right)$,

whose applicability is limited to low particle density $\rho$, a symmetric VEOS (SVEOS) equation was obtained [1]:

$\frac{P}{k_{\mathrm{B}} T}=\rho_{0} \ln \left(\frac{\rho_{0}}{\rho^{\prime}}\right)+\rho_{0} \frac{u_{0}}{k_{\mathrm{B}} T}+$

(c) M.V. USHCATS, L.A. BULAVIN, V.M. SYSOEV, S.YU. USHCATS, 2017

ISSN 2071-0194. Ukr. J. Phys. 2017. Vol. 62, No. 6 $+\rho^{\prime}\left(1-\sum_{k \geq 1} \frac{k}{k+1} \beta_{k} \rho^{\prime k}\right)+\rho_{0} \sum_{k \geq 1} \beta_{k} \rho^{\prime k}$.

Unlike Eq. (1), the validity of this equation is limited to high-density states (the low hole density $\rho^{\prime}=$ $\rho_{0}-\rho$, where $\rho_{0}$ is the close-packing density of a lattice gas, and $u_{0}$ the energy of the close-packing state per one particle. Both Eqs. (1) and (2) also include the same mutual set of irreducible cluster integrals, $\left\{\beta_{k}\right\}$, which are related to the corresponding virial coefficients [5].

There is a rather considerable experience in calculating such irreducible integrals $\left\{\beta_{k}\right\}$, i.e. the virial coefficients, for a variety of intermolecular interaction models [6-14]. However, modern researches [3, 15-18] testify that, at subcritical temperatures, the limit of applicability of VEOS (1) is a point, where the isothermal bulk modulus of the system vanishes,

533 
i.e. the value of density $\rho_{C}$ is determined by the equation

$\sum_{k \geq 1} k \beta_{k} \rho_{C}^{k}=1$

The quantity $\rho_{C}$ also determines the maximum hole density $\rho^{\prime}$ (and the corresponding minimum density $\rho=\rho_{0}-\rho^{\prime}$ ), at which SVEOS (2) remains valid.

In work [2], the same "particle-hole" symmetry was used to consider another pair of symmetric equations of state in the form of power series expansions of the pressure and density in the activity $z$ [this quantity is associated with the chemical potential $\mu$ and the de Broglie wavelength of molecules $\lambda$ by the formula $\left.z=\lambda^{-3} \exp \left(\mu / k_{\mathrm{B}} T\right)\right]$ :

$$
\left.\begin{array}{l}
\frac{P}{k_{\mathrm{B}} T}=\sum_{n=1}^{\infty} b_{n} z^{n} \\
\rho=\sum_{n=1}^{\infty} n b_{n} z^{n}
\end{array}\right\},
$$

and

$$
\left.\begin{array}{l}
\frac{P}{k_{\mathrm{B}} T}=\rho_{0}\left(\frac{u_{0}}{k_{\mathrm{B}} T}+\ln \frac{\rho_{0}}{\eta}\right)+\sum_{n \geq 1} b_{n} \eta^{n} \\
\rho=\rho_{0}-\sum_{n \geq 1} n b_{n} \eta^{n}
\end{array}\right\},
$$

where

$\eta=\frac{\rho_{0}^{2}}{z} \exp \left(\frac{2 u_{0}}{k_{\mathrm{B}} T}\right)$

is the quantity reciprocal to the activity.

The coefficients $b_{n}$ in Eqs. (4) and (5) are the socalled reducible cluster integrals [5], which are exactly expressed, provided an unconfined integration volume, in terms of irreducible ones,

$b_{n}=n^{-2} \sum_{\left\{j_{k}\right\}} \prod_{k=1}^{n-1} \frac{\left(n \beta_{k}\right)^{j_{k}}}{j_{k} !}$,

where $\left\{j_{k}\right\}$ are all possible sets of $k$ numbers that satisfy the condition

$\sum_{k=1}^{n-1} k j_{k}=n-1$.
The series expansions of the pressure and density in Eqs. (4) and (5) directly express the logarithm of the grand partition function and its derivative with respect to the chemical potential. Therefore, they have a more rigorous statistical substantiation in comparison with the virial series expansions (1) and (2); unlike the VEOS, they were obtained on the basis of the Mayer cluster expansion [5] without additional restrictions on the density. Unfortunately, the usage of such parametrical equations remained to be connected for a long time with certain mathematical and technical difficulties, so that it was VEOS (1) that obtained a wider practical application.

On the one hand, the series for the pressure and density in Eq. (4) can diverge in dense states (with high activity values) $[19,20]$. On the other hand, researches of the behavior of high-order terms in those series remain almost impossible because of an extreme complexity of practical applications of relation (6) together with condition (6a). For example, in work [21], by expanding the van der Waals equation in a power series in the density, the behavior of the equation of state with reducible cluster integrals was analyzed, but only for systems with very small dimensions. In work [2], the reducible cluster integrals for a lattice gas in the Lee-Young model [22] were calculated only to the seventh order, and a comparison with the behavior of the corresponding VEOS and SVEOS showed a considerable difference even for densitys much lower that $\rho_{C}$. For an adequate comparison to be done between the behaviors of series expansions in powers of the density [Eqs. (1) and (2)] and activity [Eqs. (4) and (5)] - and, even more so, for the research of the series divergence in Eqs. (4) and (5) - the latter should include much larger number of terms $(n \rightarrow \infty)$ : the VEOS and SVEOS, even with the finite $\left\{\beta_{k}\right\}$ set, correspond to power expansions in the activity with an unconfined set of all reducible integrals $\left\{b_{n}\right\}$, which can be determined, in general, in Eq. (6) on the basis of the same finite set of irreducible integrals $\left\{\beta_{k}\right\}$.

In this work, a more convenient, in comparison with formula (6), relation between the reducible, $\left\{b_{n}\right\}$, and irreducible, $\left\{\beta_{k}\right\}$, cluster integrals is considered (Section 2). In Section 3, this relation is used to study the behavior of expansions (4) and (5) with regard for high-order reducible integrals, in particular, the character of their divergence at large $z$ and $\eta$, respectively.

ISSN 2071-0194. Ukr. J. Phys. 2017. Vol. 62, No. 6 


\section{Algorithm for the Calculation of Reducible Cluster Integrals}

Mathematically, Eqs. (6) and (6a) mean that the quantity $n^{2} b_{n}$ is a coefficient in front of $x^{n-1}$ in the series expansion of the function

$F(x)=\exp \left(n \sum_{k=1}^{\infty} \beta_{k} x^{k}\right)$

i.e.

$n^{2} b_{n}=\frac{1}{(n-1) !}\left(\frac{\partial^{n-1} F}{\partial x^{n-1}}\right)_{x=0}$.

Writing down function (7) in the form

$F(x)=\exp [f(x)]$,

where

$f(x)=n \sum_{k=1}^{\infty} \beta_{k} x^{k}$,

one can see that its derivative of the $i$-th order is determined by the following recursive relation:

$F^{(i)}=\frac{\partial^{i} F}{\partial x^{i}}=\sum_{k=1}^{i} \frac{(i-1) !}{(k-1) !(i-k) !} f^{(k)} F^{(i-k)}$,

where $F^{(0)}$ means the function $F(x)$ itself, and $f^{(k)}$ is the derivative of the $k$-th order of the internal function $f(x)$,

$f^{(k)}=\frac{\partial^{k} f}{\partial x^{k}}=n \sum_{m=k}^{\infty} \frac{m !}{(m-k) !} \beta_{m} x^{m-k}$.

The values of those derivatives at the point $x=0$ equal

$f_{0}^{(k)}=\left.f^{(k)}\right|_{x=0}=n \cdot k ! \beta_{k}$,

and

$F_{0}^{(i)}=n \sum_{k=1}^{i} \frac{(i-1) !}{(i-k) !} k \beta_{k} F_{0}^{(i-k)}$,

where $F_{0}^{(0)}=F(0)=1$.

Let us introduce the notation

$B_{i}=\frac{F_{0}^{(i)}}{i !}$.

ISSN 2071-0194. Ukr. J. Phys. 2017. Vol. 62, No. 6
Then Eq. (9) is transformed into the formula

$B_{i}=n \sum_{k=1}^{i} \frac{k}{i} \beta_{k} B_{i-k}$,

where $B_{0}=1$.

According to Eq. (8),

$n^{2} b_{n}=\frac{F_{0}^{(n-1)}}{(n-1) !}=B_{n-1}$

or

$b_{n}=\frac{B_{n-1}}{n^{2}}$.

From the mathematical viewpoint, such a definition of an arbitrary reducible cluster integral $b_{n}$ (11) in terms of irreducible cluster integrals $\left\{\beta_{k}\right\}$ with the use of the recursive relation (10) remains to be identical to Eq. (6) with condition (6a). At the same time, it is much more convenient and makes it possible to efficiently perform numerical calculations of reducible cluster integrals even of very high orders.

\section{Character of Divergence in the Power Expansions in the Activity}

The behavior of the pressure and density expansions in Eqs. (4) and (5) was studied, by using the specific model of a lattice gas as an example, for which the exact values of gas-liquid phase transition parameters, namely, the pressure $P_{0}$ and the densities at the boiling point, $\rho_{L}$, and dry saturated vapor point, $\rho_{G}$, are known [22]:

$$
\left.\begin{array}{l}
\frac{P_{0}}{\rho_{0} k_{\mathrm{B}} T}=\ln (1+a)+\frac{1}{2 \pi} \int_{0}^{\pi} \ln \left(\frac{1+\sqrt{1-a b^{2} \sin ^{2} \varphi}}{2}\right) d \varphi \\
\rho_{L}=\rho_{0}-\rho_{G} \\
\rho_{G}=\frac{\rho_{0}}{2}\left(1-\sqrt{\frac{\sqrt{(1+a) \sqrt{1-6 a+a^{2}}}}{1-a}}\right)
\end{array}\right\}
$$

where

$a=\exp \left(-\frac{\varepsilon}{k_{\mathrm{B}} T}\right), \quad b=4 \frac{1-a}{(1+a)^{2}}$.

In this two-dimensional Lee-Young model, absolutely hard particles attract only those particles that 


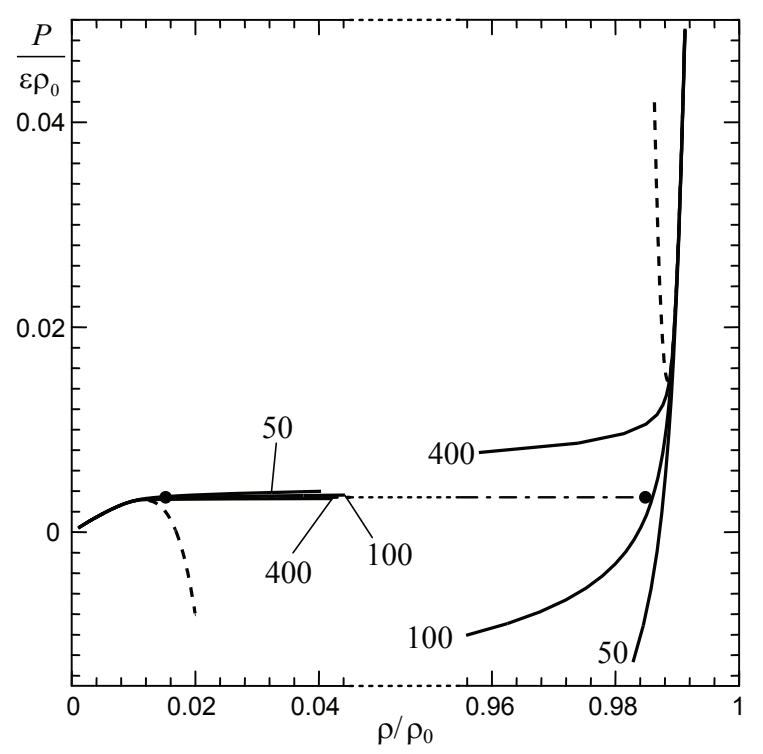

Fig. 1. Isotherms of Eqs. (4) (on the left) and (5) (on the right) (the maximum order of reducible integrals is indicated), and VEOS (1) and SVEOS (2) (the corresponding dashed curves) for the Lee-Young lattice gas at the subcritical temperature $T=0.4 \varepsilon / k_{\mathrm{B}}$ with regard for the first five irreducible integrals. The horizontal dash-dotted segment corresponds to the Lee-Young solution (12)

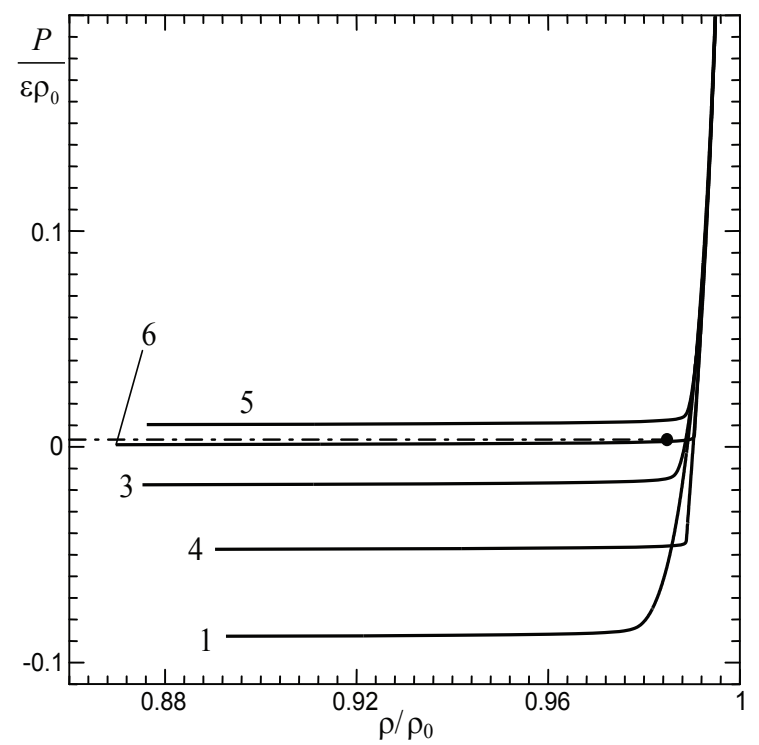

Fig. 2. Isotherms of Eq. (5) with reducible integrals to the 800-th order for the Lee-Young lattice gas at the subcritical temperature $T=0.4 \varepsilon / k_{\mathrm{B}}$ with regard for different numbers of irreducible integrals (the maximum order is indicated). The horizontal dash-dotted segment corresponds to the Lee-Young solution (12) are located in four neighbor cells of a square lattice (the square-well potential with the depth $\varepsilon$ ), and the energy per one particle in the close-packing state equals $u_{0}=-2 \varepsilon$ [this parameter enters Eqs. (2) and (5)].

For this model, the irreducible cluster integrals $\left\{\beta_{k}\right\}$ were calculated in work [2] up to the sixth order (the virial coefficients to the seventh order). On the basis of this information and with the use of the algorithm presented in the previous section, we calculated the corresponding reducible cluster integrals $\left\{b_{n}\right\}$ in order to use them in Eqs. (4) and (5).

Figure 1 demonstrates the VEOS [Eq. (1)] and SVEOS [Eq. (2)] isotherms obtained with regsrd for the first five irreducible integrals $\left\{\beta_{1}, \beta_{2}, \beta_{3}, \beta_{4}, \beta_{5}\right\}$, as well as the isotherms of Eqs.(4) and (5), which include the reducible cluster integrals $\left\{b_{n}\right\}$ of various orders calculated on the basis of the same set. In the regions where the VEOS and SVEOS are adequate ( $\rho<\rho_{C}$ and $\rho^{\prime}<\rho_{C}$, respectively), the isotherms of virial power expansions in the density [Eqs. (1) and (2)] and activity [Eqs. (4) and (5)] have expectedly a good convergence. But beyond the zero point of the isothermal bulk modulus $\rho_{C}$, when it is exceeded by the particle density $\rho$ in Eqs. (1) and (4), and by the hole density $\rho^{\prime}$ in Eqs. (2) and (5), the behaviors of those expansions start to differ substantially (see Fig. 1). With the growth of the order of expansions in the activity [Eqs. (4) and (5)], their isotherms become more planar in this region rather than approach the isotherms of the corresponding VEOS (1) and SVEOS (2).

On the one hand, very rapid variations of the density, when the activity $z$ in Eq. (4) or the reciprocal activity $\eta$ in Eq. (5) approaches a certain value corresponding to the density $\rho_{C}$ in Eq. (3), testify to the divergence of Eqs. (4) and (5) in a vicinity of the point $\rho_{C}$. Furthermore, the rate of divergence of the density expansions (i.e. the rate of density variation with the activity growth) in those equations should considerably exceed the rate of divergence of the corresponding pressure expansions, which results in the observable pressure constancy along the isotherms.

On the other hand, such a behavior agrees well with the results of modern researches dealing with the cluster expansion of the partition function in terms of the density $[3,15-18]$, where the dependence on the activity was excluded. First, the inadequacy of VEOS (and SVEOS) beyond the zero point of the isothermal 
bulk modulus $\rho_{C}$ was confirmed. Second, the pressure invariance along the isotherms points to the condensation in the corresponding region.

It should be noted that a similar behavior is observed, if various numbers of irreducible integrals (virial coefficients) are taken into account. Unfortunately, the limitation of the known coefficient set results in a still appreciable difference between the results of calculations and the Lee-Young solution (12). However, with the extension of this set, the isotherms gradually (although in a complicated way) come closer to the exact solution (see Fig. 2).

In addition, at the qualitative level, the mathematical divergence of the series expansions in Eqs. (4) and (5) corresponds to thermodynamic ideas about the first-order phase transitions and, in particular, to the Maxwell rule; namely, the density is changed against the practically constant values of pressure and activity (i.e. the chemical potential).

\section{Conclusions}

The recursive relation between the reducible and irreducible cluster integrals on the basis of Eqs. (10) and (11) is much more efficient than the convensional relation (6). In particular, it allows the reducible integrals $\left\{b_{n}\right\}$ of rather high orders (hundreds and even thousands) to be calculated on the basis of any known set of irreducible integrals $\left\{\beta_{k}\right\}$ (virial coefficients). This relation allows comprehensive researches of the behavior of the well-known equation of state (4) to be carried out in the form of the pressure and density expansions in the powers of activity with regard for the terms of very high orders, which was earlier associated with considerable difficulties.

In particular, for the known Lee-Young model of a lattice gas [22], the behaviors of Eq. (4) together with the recently obtained symmetric equation of state (5) were studied in the region, where the validity of the corresponding virial power expansions in the density [Eqs. (1) and (2)] becomes doubtful. Notwithstanding a very limited set of known irreducible integrals (virial coefficients) and appreciable quantitative deviations of the calculated isotherms from the exact solution (12), our researches allow a number of important conclusions to be drawn at a qualitative level.

First of all, the applicability of the well-known VEOS (1), which was obtained from the very begin- ning as a replacement of Eq. (4) with the purpose to avoid the problems associated with the divergence of its series expansion in the activity $[4,5,20]$, turns out to be confined to the region, where those series converge, namely, by the point $\rho_{C}[$ Eq. (3)], where the isothermal bulk modulus vanishes, which quite agrees with the results of modern researches concerning the cluster expansion of a partition function in terms of the density [3,15-18]. The same is valid for the symmetric equations (2) and (5) for the dense states of a lattice gas: SVEOS (2) is applicable only in the convergence region of the more general equation (5).

The expansions of the pressure and density in the power series of activity in Eqs. (4) and (5) with regard for terms of high orders directly demonstrate a divergence in a vicinity of the point $\rho_{C}$. However, the character of this divergence does not testify to the mathematical inadequacy of the cluster expansion of the partition function (as was considered earlier [4]), but, on the contrary, has a rather clear physical sense corresponding to the convensional thermodynamic ideas about the gas-liquid phase transition, namely, the density of the system is changed at the constant values of temperature, pressure, and chemical potential.

Hence, our research of the actual behavior of power expansions in the activity with a considerable number of terms confirms qualitatively the assumption of some researchers $[19,20]$ about the connection between the condensation and the divergence of those expansions. Again, this result agrees well with the results of other researches [3, 15-18,21], which identify the density $\rho_{C}$ as the condensation point (a binodal point).

Moreover, the observed behavior of Eqs. (4) and (5) - which, in essence, are nothing else but the logarithm of the grand partition function (the series for pressure) and its derivative with respect to the chemical potential (the series for density) - gives a possible statistical explanation to the condensation process (and the inverse boiling process). Namely, the mathematical divergence of the series means a principal increase of the contribution made by high-order terms to the total sum (i.e. the growth of the statistical weight of corresponding high-order reducible integrals in the partition function) and therefore a qualitative growth of the probability for large clusters to exist. For Eq. (4), this means the appearance 
of liquid droplets (large clusters of particles) in the gas phase, i.e. the beginning of the condensation. For Eq. (5), this means the appearance of gas bubbles (large clusters of "holes") in the liquid phase, i.e. the beginning of the boiling.

1. M.V. Ushcats. High-density equation of state for a lattice gas. Phys. Rev. E 91, 052144 (2015).

2. M.V. Ushcats, L.A. Bulavin, V.M. Sysoev, S.J. Ushcats. Virial and high-density expansions for the Lee-Yang lattice gas. Phys. Rev. E 94, 012143 (2016).

3. M.V. Ushcats, L.A. Bulavin, V.M. Sysoev, V.Y. Bardik, A.N. Alekseev. Statistical theory of condensation - Advances and challenges. J. Mol. Liq. 224, 694 (2016).

4. R. Balescu. Equilibrium and Nonequilibrium Statistical Mechanics (Wiley, 1975) [ISBN: 0471046000].

5. J.E. Mayer, M.G. Mayer. Statistical Mechanics (Wiley, 1977)

6. C. Feng, A.J. Schultz, V. Chaudhary, D.A. Kofke. Eighth to sixteenth virial coefficients of the Lennard-Jones model. J. Chem. Phys. 143, 044504 (2015).

7. M.V. Ushcats. Modified Lennard-Jones model: Virial coefficients to the 7th order. J. Chem. Phys. 140, 234309 (2014).

8. M.V. Ushcats. Communication: Low-temperature approximation of the virial series for the Lennard-Jones and modified Lennard-Jones models. J. Chem. Phys. 141, 101103 (2014).

9. A.J. Schultz, D.A. Kofke. Vapor-phase metastability and condensation via the virial equation of state with extrapolated coefficients. Fluid Phase Equilibria 409, 12 (2016).

10. M.V. Ushcats. Virial coefficients of modified LennardJones potential. Ukr. Fiz. Zh. 59, 172 (2014).

11. M.V. Ushcats. Modification of the Mayer sampling method for the calculation of high-order virial coefficients. Ukr. Fiz. Zh. 59, 737 (2014).

12. M.V. Ushcats, S.J. Ushcats, A.A. Mochalov. Virial coefficients of Morse potential. Ukr. J. Phys. 61, 160 (2016).

13. M.V. Ushcats, A.A. Gaisha. The fifth virial coefficient for the Sutherland, Morse, and Lennard-Jones potentials. Visn. Kharkiv Nat. Univ. 18, 67 (2013) (in Russian).
14. M.V. Ushcats, K.D. Evfimko. The sixth virial coefficient for the modified Lennard-Jones potential, Visn. Odessa Nat. Univ. 19 (1), 37 (2014).

15. M.V. Ushcats. Equation of state beyond the radius of convergence of the virial expansion. Phys. Rev. Lett. 109, 040601 (2012).

16. M.V. Ushcats. Visn. Kharkiv Nat. Univ. 17, 6 (2012).

17. M.V. Ushcats. Adequacy of the virial equation of state and cluster expansion. Phys. Rev. E 87, 042111 (2013).

18. M.V. Ushcats. Condensation of the Lennard-Jones fluid on the basis of the Gibbs single-phase approach. J. Chem. Phys. 138, 094309 (2013).

19. J. Groeneveld. Two theorems on classical many-particle systems. Phys. Lett. 3, 50 (1962).

20. O. Penrose. Convergence of fugacity expansions for fluids and lattice gases. J. Math. Phys. 4, 1312 (1963).

21. E. Donoghue, J.H. Gibbs. Condensation theory for finite, closed systems. J. Chem. Phys. 74, 2975 (1981).

22. T.D. Lee, C.N. Yang. Statistical theory of equations of state and phase transitions. II. Lattice gas and Ising model. Phys. Rev. 87, 410 (1952).

Received 11.04.17.

Translated from Ukrainian by O.I. Voitenko

М.В. Уикаи, Л.А.Булавін, В.М. Сисоєв, С.Ю. Уикаи,

КОНДЕНСАЦІЯ ГРАТКОВОГО ГАЗУ

ТА ÏÏ ЗВ'ЯЗОК З РОЗБІЖНІСТЮ ВІРІАЛЬНИХ

РОЗКЛАДІВ ЗА СТЕПЕНЯМИ АКТИВНОСТІ

$\mathrm{P}$ е $з$ ю м е

Представлено ефективний алгоритм розрахунку звідних групових інтегралів високих порядків на основі набору незвідних інтегралів (віріальних коефіцієнтів). За його допомогою для специфічної моделі граткового газу була досліджена поведінка відомих віріальних розкладів тиску й густини за степенями активності та нещодавно отриманих симетричних розкладів за степенями оберненої активності 3 урахуванням доданків дуже великих порядків. Отримані результати узгоджуються з іншими сучасними дослідженнями статистичної суми в термінах густини та розкривають фізичний зміст розбіжності вказаних розкладів в області конденсації. 